\title{
Una Aproximación Relacional a la Violencia Escolar Entre Pares en Adolescentes Chilenos: Perspectiva Adolescente de los Factores Intervinientes
}

\section{A Relational Approach to School Violence Among Peers in Chilean Adolescents: Adolescent Perspective About Intervening Factors}

\author{
Maritgen Potocnjak \\ Universidad Alberto Hurtado
}

\author{
Christian Berger \\ Pontificia Universidad Católica de Chile
}

\author{
Tatiana Tomicic \\ Universidad Alberto Hurtado
}

\begin{abstract}
El presente estudio aborda la violencia escolar desde la perspectiva de los adolescentes, identificando factores intervinientes en su emergencia. Se utilizó una metodología cualitativa exploratoria enmarcada en la grounded theory, realizando entrevistas individuales y grupales a una muestra intencionada de 28 estudiantes de 11 a 14 años de 3 colegios de la Región Metropolitana de Chile. Los resultados evidencian un distanciamiento entre las lógicas adultas y las necesidades socioafectivas de los estudiantes, lo que favorecería el involucramiento en violencia escolar. La escuela y los profesores no tendrían estrategias efectivas para hacer frente a este fenómeno. Las dinámicas de pares pueden sostener, potenciar o inhibir la violencia, al considerar elementos como el posicionamiento social y las jerarquías internas de la ecología social. El análisis relacional destaca la demanda adolescente por estructura, contención y orientación, planteando que el sistema adulto no satisface de manera adecuada estas necesidades. La violencia cumpliría la función de llamar la atención de los adultos y llenar el espacio de apego social y emocional que se constituye en una necesidad fundamental en esta etapa.
\end{abstract}

Palabras clave: violencia escolar, apego secundario, relación entre pares

\begin{abstract}
The present study approaches school violence from the adolescents' perspective, identifying intervening factors in its emergence. An exploratory qualitative methodology within a grounded theory framework was used, interviewing individually and collectively 28 students of 11 to 14 years old from 3 schools in the Metropolitan Region of Chile. Results show a gap between adult perspectives and students' socioemotional needs, which would favor their involvement in school violence. Schools and teachers do not have effective strategies to face this phenomenon. Peer dynamics can sustain, reinforce, or inhibit violence, through the establishment of social hierarchies within the peer social ecology. The relational analysis highlights the adolescent demands for structure, contention, and orientation, arguing that the adult system does not satisfy these needs adequately. Adolescents would build through violence a social order aimed to meet their relational needs. Violence might fulfill the function of calling adults' attention and fill the space of the social and emotional attachment dimension which constitutes a central need during this developmental stage.
\end{abstract}

Keywords: school violence, secondary attachment, peer relations

Maritgen Potocnjak, Facultad de Psicología, Universidad Alberto Hurtado, Santiago, Chile; Christian Berger, Escuela de Psicología, Pontificia Universidad Católica de Chile, Santiago, Chile; Tatiana Tomicic, Facultad de Psicología, Universidad Alberto Hurtado, Santiago, Chile.

Este artículo es parte del trabajo de tesis de la primera autora para optar al grado de Magíster en Psicología de la Universidad Alberto Hurtado.

La correspondencia relativa a este artículo debe ser dirigida a Maritgen Potocnjak, Facultad de Psicología, Universidad Alberto Hurtado, Almirante Barroso 26, Santiago, Chile. E-mail: maritgen_potocnjak@yahoo.es 
En las últimas décadas la temática de la violencia en contextos escolares ha cobrado gran relevancia. Si bien se han realizado estudios sistemáticos sobre el tema de violencia entre pares en contexto escolar a nivel mundial (para una revisión ver Jimerson, Swearer \& Espelage, 2010), dichas investigaciones han sido principalmente de corte cuantitativo y se han centrado en describir el fenómeno, su prevalencia y los factores asociados (Almeida, Lisboa \& Caurcel, 2007; Berger, Karimpour \& Rodkin, 2008; Orpinas \& Horne, 2006; Sullivan, Cleary \& Sullivan, 2005). Sin embargo, existe menos información sobre la subjetividad de los mismos involucrados respecto de la violencia. Esta información es particularmente relevante para identificar los factores involucrados en el desarrollo de la violencia escolar, cuya evaluación y modificación aparece como una fórmula fundamental para generar estrategias de prevención e intervención (Organización Panamericana de la Salud \& Cooperación Técnica Alemana-GTZ, 2008; Orpinas \& Horne, 2006).

En Chile el interés por conocer y comprender la violencia escolar ha sido rápido y creciente, pero se ha abordado públicamente recién a partir de la última década. Durante el año 2005, conjuntamente con el Ministerio del Interior y su política de seguridad ciudadana, el Ministerio de Educación promueve la implementación de la Primera Encuesta Nacional de Violencia en el Ámbito Escolar. En la misma encuesta en su tercera versión se señala que un 23,3\% de los escolares reconocen haber sido agredidos en su establecimiento educacional (Chile, Ministerio del Interior, 2011). Si bien estas cifras son preocupantes, evidencian que la mayoría de los alumnos y alumnas no se involucran en dinámicas violentas. El último estudio nacional señala que un 60,9\% de los entrevistados declara que no es ni agresor ni agredido (Chile, Ministerio del Interior, 2011). Precisamente este aspecto es el punto de partida del presente estudio: identificar los factores que, según la perspectiva de los propios estudiantes, inhiben o favorecen el involucramiento en situaciones de violencia escolar entre pares. Se adoptó una mirada ecológica (Bronfenbrenner, 1979), comprendiendo el desarrollo individual en el marco de contextos sociales con grados crecientes de complejidad, y un enfoque conceptual basado en la psicología relacional, focalizando los aspectos vinculares asociados a la emergencia, mantención e inhibición de dinámicas de violencia entre pares.

\section{Violencia Escolar}

Una dificultad para investigar sobre violencia escolar es su definición. De hecho, generalmente se utiliza indistintamente el término bullying como sinónimo (Del Barrio, Martín, Almeida \& Barrios, 2003). Como destacan estos autores, las dinámicas de bullying se refieren a dinámicas de maltrato, o bien de abuso, que se fundamentan en cuatro características específicas: que sea entre pares, que sea constante en el tiempo, que implique un desequilibrio de poder y que constituya una experiencia de victimización (Olweus, 1997; Orpinas \& Horne, 2006). Sin embargo, otras conductas de violencia no se dan en el contexto de una relación de abuso, siguiendo la descripción anterior. La violencia puede ser significada como una forma de relación interpersonal, de convivencia, y no exclusivamente como una forma de maltrato. Lo que configura a un acto como violencia entre pares, desde la perspectiva de los actores, es la experiencia de violencia, victimización y daño, pero no la conducta en sí (Berger \& Rodkin, 2009; Graham \& Juvonen, 1998). Por ejemplo, Troop-Gordon y Ladd (2005) muestran que hombres y mujeres pueden interpretar de maneras distintas su experiencia de victimización. En Chile Contador (2001) encontró diferencias de género y edad en la percepción y la vivencia emocional de la violencia. Del Barrio, Almeida, Van der Meulen, Barrios y Gutiérrez (2003) reportaron diferencias tanto de género como según la edad de estudiantes españoles y portugueses, observando, además, diferencias según el rol o perspectiva que adoptaban los participantes para describir situaciones de violencia. Asimismo, Smith, Cowie, Olafsson y Liefooghe (2002) mostraron en su estudio comparativo que la violencia entre pares es significada y señalada de maneras muy distintas en diferentes países y grupos etarios. Frente a esta dificultad, algunos autores han optado por no ofrecer una definición a priori del fenómeno a estudiar (Espelage, Holt \& Henkel, 2003; García \& Madriaza, 2005) y observar la construcción que los propios actores de la violencia hacen del fenómeno.

En este contexto, Berger y Lisboa (2008) conceptualizan la violencia entre pares como un proceso, resultado de la interacción entre las características individuales con sus contextos. 
Como señalan Del Barrio, Martín et al. (2003, p. 12), “estos comportamientos (...) solo es posible entenderlos como fenómenos de grupo, que no ocurrirían fuera del mismo". Considerando lo anterior, el foco de este estudio se orienta a la violencia como una forma de relación interpersonal y no al maltrato en el sentido de dinámicas de abuso. Si bien todo acto de violencia implica inherentemente un acto de abuso, interesa especialmente su carácter funcional dentro de la cultura de pares adolescentes. Desde esta perspectiva, la violencia escolar debe ser comprendida más allá de las particularidades individuales de quienes participan de ella, constituyéndose en un fenómeno interpersonal y grupal (Del Barrio, Martín et al., 2003), determinado por todas las interacciones significativas entre personas, objetos y símbolos en un ambiente inmediato y en un determinado espacio temporal.

\section{Perspectiva Vincular del Desarrollo y Violencia}

Tanto desde la teoría del aprendizaje como desde las teorías intersubjetivas se ha considerado que los vínculos afectivos tempranos sanos constituyen la base de un desarrollo saludable (Benjamin, 1988; Gallese, Eagle \& Migone, 2006). La necesidad de contar con una figura a quien apegarse, que entregue una base personal segura, no es exclusiva de los niños, sino que se mantiene en adolescentes y adultos (Bowlby, 1999; Roisman, Padron, Sroufe \& Egeland, 2002; Scharf \& Mayseless, 2007). La mayoría de los autores especializados en violencia escolar coincide en que los vínculos con los padres que son nutritivos, sintonizados con las necesidades infantiles, no agresivos y estables disminuyen la probabilidad de que los niños presenten problemas conductuales o comportamientos violentos (Bergin \& Bergin, 2009; Olweus, 1997; Orpinas \& Horne, 2006).

En la adolescencia el desarrollo de la identidad pone en tensión los cimientos afectivos del joven y, en consecuencia, su historia de apego. Aun cuando en la adolescencia el apego se actualiza fuertemente a través de las relaciones de grupo que pasan a ser una parte fundamental de la consolidación de la identidad que se genera en este estadio, la familia y los adultos significativos continúan siendo parte importante del proceso de cambio y crecimiento. Las personas se desarrollan en el marco de sus vínculos, estableciéndose el self en lo interpersonal, manteniéndose y reorganizándose a través de transacciones interpersonales durante el ciclo vital (Shapiro, 1991).

García y Madriaza (2005) proponen que la agresión para los niños y jóvenes tiene un carácter simbólico que se relaciona con el reconocimiento por parte de los pares y, así, con la construcción de la identidad en la etapa adolescente. Como señalan los autores:

El golpe, al poner en juego los límites corporales, permite establecer la frontera personal en relación al otro. Se podría pensar que la violencia no física funciona también como una forma de instaurar esta identidad personal y, por supuesto, también la del otro. (García \& Madriaza, 2005, p. 24)

La violencia también puede cumplir una función adaptativa dentro de la cultura de pares; en la medida que las normas grupales validen la violencia como una forma de relación con otros, ser violento favorece la integración al grupo a través del cumplimiento del perfil deseado (Berger \& Rodkin, 2011). Precisamente, lo anterior explica en parte que la edad de mayor prevalencia de dinámicas de violencia sea entre los 11 y 14 años (Berger et al., 2008; Chile, Ministerio del Interior, 2011; Sullivan et al., 2005), etapa en la que es una tarea evolutiva consolidar un lugar propio en el grupo de pares en relación al desarrollo de la identidad. De esta forma, los vínculos interpersonales, y más aún las figuras significativas que favorezcan una buena integración de dichos vínculos, pueden jugar un importante rol en las dinámicas de violencia.

A la luz de los antecedentes teóricos y empíricos antes expuestos, resulta evidente la necesidad de ampliar la comprensión de la violencia escolar entre pares desde una mirada relacional y evolutiva. Más aún, la consideración de la perspectiva de los propios involucrados resulta esencial para acceder a los significados, creencias y subjetividad que estos tienen respecto del fenómeno. En concreto, el presente estudio tuvo por objetivo identificar los factores que los propios estudiantes identifican como protectores o facilitadores de la violencia escolar en los diferentes contextos que configuran su experiencia cotidiana. 


\section{Método}

El presente estudio adoptó una metodología de investigación cualitativa, utilizando el enfoque de la grounded theory (Glaser \& Strauss, 1967; Strauss \& Corbin, 2002), el cual es definido como un método que pretende construir teorías explicativas de fenómenos sociales a partir del levantamiento de datos empíricos que son sistemáticamente analizados durante el proceso de investigación. A partir de las experiencias subjetivas de los participantes de la investigación, se accede al significado de la acción social y se desarrolla un conjunto de conceptos que, al integrarse, proveen una explicación teórica-descriptiva del fenómeno estudiado (Strauss \& Corbin, 2002). Como este estudio se centró en la experiencia subjetiva de los actores, se decidió no ofrecer una definición a priori del fenómeno a estudiar. Por el contrario, la definición quedó abierta a ser conceptualizada por los adolescentes que participaron en la investigación, como manera de contextualizar su mirada y facilitar su propio discurso.

\section{Participantes}

La muestra fue intencionada e incluyó a 28 preadolescentes (14 mujeres) entre 11 y 14 años (distribuidos equitativamente por edad), pertenecientes a tres colegios particulares subvencionados de distintos sectores (poniente, oriente y sur) de la Región Metropolitana de Chile. Los criterios para el muestreo fueron la edad (la mayor prevalencia de la violencia escolar entre pares se ubica en este rango) y el tamaño del establecimiento (se seleccionaron establecimientos de distinto tamaño en base a las primeras entrevistas realizadas).

\section{Instrumentos}

La información fue recolectada por medio de dos dispositivos de carácter cualitativo: entrevistas grupales y entrevistas individuales semi-estructuradas en profundidad. Para ambos dispositivos se utilizó un guión de preguntas que fue desarrollado y piloteado previamente a la investigación. Su foco central buscó indagar en: ¿qué factores piensan (creen) que influyen o afectan en el hecho de que los estudiantes se involucren (o no se involucren) en violencia escolar entre compañeros o pares? En total se realizaron seis grupos focales con cuatro estudiantes cada uno (dos en cada establecimiento: un grupo de 11 y 12 años y otro de 13 y 14 años, respectivamente, de dos hombres y dos mujeres cada uno), con el fin de facilitar la conversación entre ellos. El guión se adaptó a las edades de los participantes. Las entrevistas individuales (cuatro) se realizaron con estudiantes de distintos colegios, incluyendo a dos mujeres (11 y 13 años) y dos hombres (12 y 13 años). Todas las entrevistas fueron grabadas y luego transcritas verbatim para su posterior codificación. En los grupos focales se cuidó de que no hubiesen conflictos previos entre participantes de un mismo grupo.

\section{Análisis de la Información}

La codificación y análisis de los datos siguió las tres instancias propuestas por la grounded theory: una codificación abierta, realizándose un análisis descriptivo de la información; una codificación axial con un análisis relacional de la información; y la codificación selectiva, con la construcción del modelo o teoría explicativa. La codificación de los datos en sus distintas etapas fue realizada y triangulada por los autores del artículo, para evitar sesgos.

\section{Procedimiento}

Se obtuvo el consentimiento de los padres, la autorización del colegio, además del asentimiento informado de los propios participantes, explicitando su carácter voluntario, confidencial y anónimo. Se garantizó contención, acogida e intervención en caso de ser requerida, situación que no se presentó. 


\section{Resultados}

A continuación se presentan los resultados obtenidos, siguiendo el procedimiento de la grounded theory. En primer lugar, se describen las categorías emergentes identificadas en el relato de los participantes. Luego, se presenta el análisis relacional, identificando como fenómeno emergente las dinámicas relacionales de los actores de la violencia escolar. Finalmente, se presenta la codificación selectiva, en la cual se identifica y desarrolla el fenómeno nuclear que permite dar sentido a los resultados: la demanda de rol adulto de afecto, protección y formación. Para ejemplificar los resultados se incluyen extractos literales del relato de los participantes.

\section{Análisis Descriptivo}

Violencia como fenómeno negativo. La totalidad de los preadolescentes entrevistados coinciden en valorar negativamente el fenómeno, tanto por sus motivaciones como por sus consecuencias. Tienden principalmente a construir un discurso desde lo que han escuchado, una definición externa del fenómeno: "Por lo que dicen las noticias, que les pegan a los alumnos, la discriminación, todo eso...." (hombre, 13 años). También aparece un reconocimiento de que los juegos de video e Internet, como grabaciones de peleas entre jóvenes o blogs, ponen el tema de la violencia en el imaginario social como una forma de juego y de competencia social, como un medio para ser más reconocidos, señalando que es negativo pero que puede resultar entretenido verlo y/o jugar, pues permite lograr mayor estatus social al publicar las peleas en Internet y como modelo de imitación: "Tal vez cree que eso es bueno y que es bacán y quiere figurar igual que el otro" (hombre, 14 años).

Violencia entre pares como un fenómeno complejo. Los adolescentes manejan indistintamente los conceptos de violencia escolar, bullying y agresión. En la construcción de la noción de violencia coinciden en que esta es un maltrato físico, verbal y una manera de discriminar, pudiendo haber amenazas directas o por Internet en medios sociales. Aparece como un fenómeno normal y frecuente. Es concebido como un fenómeno grupal en el que, además de agresores y víctimas, se suman otros a agredir, defender a la víctima o ser testigo de quien gana y quien pierde: "Sí, porque primero empiezan dos y después todos se meten" (mujer, 14 años).

Un aspecto central que los adolescentes identifican en su definición de violencia es la existencia de la opción de involucrarse o no, es decir, los adolescentes reconocen una agencia en el ejercicio de la violencia, principalmente para los agresores: "Saberse comportar, poner de su parte (...). Depende de una decisión personal” (hombre, 14 años). También podrían ser las víctimas quienes eligen serlo, al no defenderse a tiempo o intentar adaptarse al contexto social de curso o colegio, como una manera de llamar la atención en el grupo.

Otra tensión importante que los adolescentes identifican en su definición de violencia es la distinción entre agresión con intención de dañar y sin intención de dañar. Esta última puede ser un juego o la manera de resolver un problema, normalmente como un evento único ("Le pego, pero no en mala onda [sin mala intención]", hombre, 12 años) y en equidad de condiciones ("Cuando se puede defender", hombre, 13 años), donde no hay consecuencias graves para ninguno, salvo casos accidentales. Por lo mismo, los vínculos de amistad pueden permanecer. Por el contrario, los estudiantes cualifican la violencia con intención de dañar al otro con propiedades específicas de mayor intensidad, mayor frecuencia, estado emocional, como rabia o envidia, consecuencias traumáticas y dinámicas específicas: "Es como con odio" (hombre, 14 años), "Le pego fuerte y se nota igual la diferencia" (mujer, 13 años). No hay equidad de condiciones, ya sea en edad o capacidad de defenderse física o psicológicamente. En este sentido, se configura como una relación de abuso más que como un conflicto interpersonal.

Género como factor cualificador de la violencia. Los adolescentes señalan que tanto hombres como mujeres participan de dinámicas de violencia, aunque identifican diferencias entre ambos géneros. A nivel intra-género las peleas son con igual intensidad entre hombres que entre mujeres, variando principalmente en el modo y frecuencia de las agresiones. Los 
entrevistados señalan que las mujeres tienden a agredir de manera más bien verbal que física, de manera más indirecta y con menor frecuencia. A nivel inter-género los estudiantes señalan que en su grupo etario ocasionalmente se agreden físicamente; los niños lo hacen más frecuentemente, aunque es mal visto, pues "los hombres tienen más fuerza" (hombre, 12 años). No obstante, a veces constituye una forma de seducción. Por su parte, expresan que las mujeres "a veces se aprovechan" (hombre, 13 años), porque en lugar de pegar, agreden verbalmente o socialmente, o descalificando.

Factores intervinientes en la violencia escolar entre pares. En primer lugar, cabe destacar que desde el relato de los adolescentes se identifican factores que pueden ser considerados de riesgo y/o protección para el involucramiento en violencia, según si se encuentran o no presentes; por lo mismo, se les denominó intervinientes. Los entrevistados identificaron factores intervinientes en diferentes dimensiones o niveles de complejidad.

A nivel individual hacen referencia a competencias socioemocionales, como la empatía: "tienen baja autocrítica, no ven que lo que hacen puede dañar, es que nadie les habla de esto, depende de que puedan conversar de estos temas" (hombre, 12 años), el autocontrol: "saber defenderse sin violencia" (hombre, 14 años) y la reflexión: "es que igual, hay que pensar las cosas antes de hacerlas, hay que ser consciente igual, es difícil, porque uno actúa así y después como que lo pienso y 'oh, la tontera que hice"' (hombre, 13 años); a características físicas asociadas a la fortaleza o debilidad y a la presencia de problemas emocionales: "Tienen un problema, de repente ya le voy a pegar por, no se po, por sentirme mejor, más alivianada" (mujer, 14 años), "Es muy distinto a los otros tipos de compañeros, es muy sensible y eso es como lo que lo molestan en realidad" (mujer, 13 años). Señalan al rendimiento escolar como un factor protector y al aburrimiento como un factor de riesgo: "De repente puede ser de aburrido, ay, estoy aburrido, vamos a molestarlo a él y así nos reímos un rato, de cómo sufre quizás" (hombre, 13 años). Destacan también la importancia de ser parte de un grupo, de tener amigos: "Si un amigo se mete en problemas..., hay que defenderlo,..., si no es que te puedes quedar como mal mirado, sin grupo" (hombre, 14 años), lo cual es relevante considerando características evolutivas de esta etapa.

Respecto de la familia, relatan que los estilos de relaciones y de ejercicio de la disciplina, así como las historias de violencia de cada familia, son factores relevantes para el involucramiento o no en violencia: "Ven que hay violencia, que se tratan a golpes (...) De repente se comportan igual en el colegio, lo sacan de ahí y van creando una mentalidad agresiva" (mujer, 13 años). "El bullying pasa porque yo creo que no tienen cariño en la casa" (mujer, 11 años), "Que los padres, que sean más responsables, que los tomen en cuenta" (mujer, 12 años). Relaciones afectivas caracterizadas por el afecto, el apoyo, la atención y la confianza serían protectoras.

A nivel de grupo de pares destacan las características del grupo de pares y su actitud hacia la violencia como un factor interviniente. Específicamente, el apoyo emocional, la lealtad y cohesión del grupo puede ser protector o de riesgo, dependiendo de la actitud del grupo.

Respecto de la institución escolar, se identifican, en primer lugar, los estilos de comunicación y el manejo de la autoridad y la disciplina como factores intervinientes; estilos más autoritarios o más permisivos serían favorecedores del involucramiento en violencia: "Falta de atención, porque en el colegio se ve que se hacen [bullying] hasta en el mismo colegio, entonces, obvio que es falta de un adulto, como alguien que sea una autoridad" (hombre, 14 años). Destacan también el cuidado afectivo hacia los estudiantes y la presencia de recursos o estrategias de intervención frente a la violencia como factores protectores.

A nivel sociocultural destacan que las campañas de prevención de la violencia y promoción de buen trato constituyen factores protectores, mientras que el manejo mediático de los casos de violencia y los estereotipos asociados a la violencia, así como la presencia de delincuencia y drogas, constituirían factores de riesgo: "Sería bueno conversar el tema en clase de orientación, deberían hacer orientación desde chiquititos” (hombre, 11 años). 


\section{Análisis Relacional: Dinámicas Relacionales de los Actores Involucrados en Violencia Escolar}

La interacción entre los distintos contextos implicados en la violencia es dinámica e involucra a todos los actores, contextos y dimensiones: “... es que [la violencia] tiene hartas cosas, tiene muchas cosas..." (hombre, 13 años); “...la sicología, la enseñanza, depende si tú peleas, depende del grupo, del profesor, de todo el lugar, de la 'fianza' [amigos protectores], de todas esas cosas" (mujer, 14 años); "Sí, todo, de la familia, de qué le enseñaron" (hombre, 13 años).

Dos grupos de actores involucrados en el fenómeno emergen del análisis relacional; estos actores o grupos evidencian la interacción de dos mundos o sistemas, un sistema adolescente y un sistema adulto. La comprensión de las relaciones de las categorías dejan al descubierto que es en el espacio relacional entre estos dos sistemas y entre distintos contextos (escolar, grupal, familiar) donde se configuran relaciones favorecedoras o inhibidoras de violencia escolar (ver Figura 1).

En el sistema adolescente se evidencia el carácter relacional de la violencia escolar entre pares. Cuando ocurre una pelea o una situación de acoso o discriminación, en el episodio van a estar involucrados activa y directamente no solo el agresor y la víctima, sino también la "fianza" (defensores), los "carboneros" (instigadores) y los testigos. La violencia permite organizar y estructurar socialmente a los adolescentes en roles que son móviles o intercambiables. Como señala una estudiante, alguien puede aparecer como víctima, pero haber sido el iniciador de la situación de violencia: "En la grabación [de video] se ve que le están pegando al pobrecito y él fue el que inició todo" (mujer, 14 años).

La organización social generada con la violencia jerarquiza a los adolescentes a través de dinámicas de poder donde se tiende a naturalizar el ejercicio de la violencia, especialmente como forma de defensa frente a las agresiones o descalificaciones. Aquel que agrede o atemoriza pasa a ser respetado, temido, admirado o victimizado, dependiendo de la asociación de una serie de elementos individuales y grupales, como las actitudes del grupo de pertenencia, la popularidad del estudiante, si adhiere a la violencia, las habilidades sociales con que cuenta, si utiliza la violencia como protección o defensa, según su apariencia física, sus logros personales o la capacidad para hacer nuevos amigos: "Yo creo que los que pelean creen que van a quedar como mejor (...). No el que tiene más fuerza, el que no se metan con él...” (mujer, 12 años). Quienes ejercen la violencia y son respetados y temidos tendrían mayores probabilidades de seguir haciéndolo, porque se les refuerza la actitud violenta; sin embargo, quienes son temidos, pero no tienen amistades o no son respetados, tienen probabilidades de continuar siendo agresores, porque no pertenecen a un grupo y buscarían la validación social, perpetuando el círculo de la violencia: "A veces lo hacen para llamar la atención, para que alguien se ría de ellos, los pesque [considere] alguien, los mire o se ría, aunque sea de ellos" (mujer, 11 años). En relación a lo anterior, surge la construcción de identidad como un elemento central para el involucramiento en violencia. La violencia se constituye en un lenguaje que confirma al otro y ayuda a la construcción de su identidad, definiendo su imagen y lugar en las interacciones diádicas y grupales.

En relación con la pertenencia grupal y la amistad, se observa la importancia de que, sea cual fuere el grupo de pertenencia y los amigos, el apoyo emocional y la protección frente a la violencia sea recíproca. Esta reciprocidad podría ser de riesgo para los agresores, porque se sienten respaldados en su conducta, pero protegería de ser víctimas, tanto física como emocionalmente: mientras mayor sea el número de amigos y "fianza" (pares protectores), menor probabilidad de que otro u otros se atrevan a agredir, pero mayor probabilidad de ser los victimarios. Así, la violencia permite conocer quiénes son los "verdaderos amigos", porque defienden activamente o apoyan psicológica y emocionalmente. Sin embargo, los jóvenes refieren que muchas veces, si no se pertenece a un grupo o no se tiene un lugar definido en el contexto social, se corre el riesgo de utilizar la violencia como un medio posicionador, ya sea como agresor o como víctima, porque en ambos roles se llama la atención. 


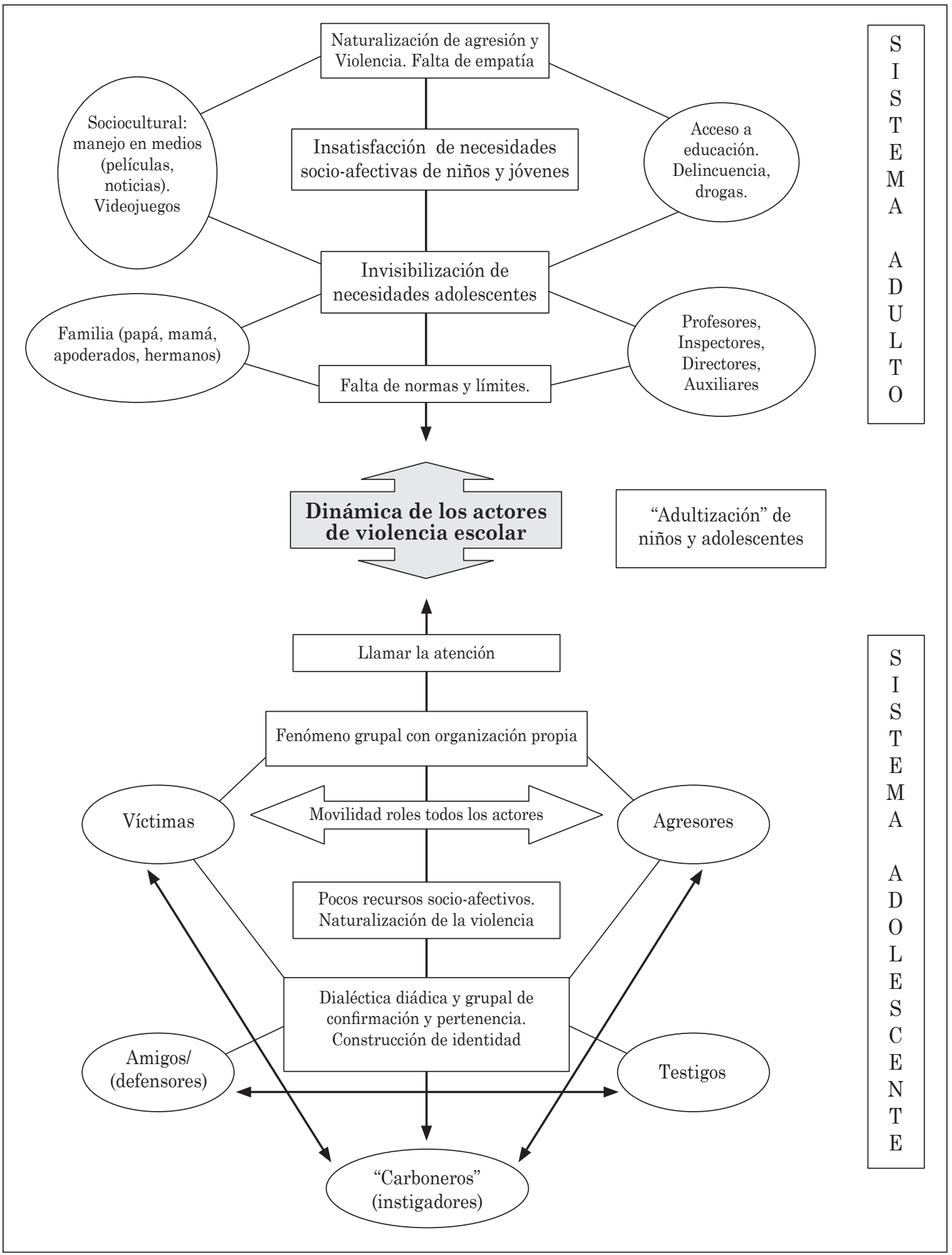

Figura 1. Dinámicas relacionales de los actores involucrados en violencia escolar. 
Respecto de las interacciones entre los sistemas adulto y adolescente, los estudiantes señalan que los adultos intervienen de un modo más bien indirecto y, en ocasiones, pasivo. Lo anterior tiene importantes implicancias para la dinámica entre ambos sistemas. En primer lugar, los adolescentes relatan la necesidad de llamar la atención de los adultos. Esto hace referencia a sus necesidades afectivas fundamentales: "Por ejemplo, porque quieren que los tomen más en cuenta en la familia y tratan de comportarse así para lograr captar la atención" (hombre, 14 años). Para los estudiantes en general los adultos no logran detectar sus necesidades de desarrollo emocional y social. Para ellos, cuando ocurren situaciones de violencia escolar, la mayoría de las veces la reacción desde los adultos hacia los adolescentes es de desinterés, poco reconocimiento y atención a sus necesidades; en sus palabras, de "no ver": "Yo el año pasado, todas las noches hablaba con ella [la mamá]. Ella llega muy cansada del trabajo y yo hablando y, de repente, la veo y estaba como quedándose dormida. Así, yo 'ah, no le hablo nada"' (mujer, 13 años).

Frente a esta necesidad, emerge del discurso de los adolescentes la adquisición de estilos adultos como una manera de organizarse, defenderse y funcionar socialmente a través de la violencia como un medio de estructuración social (“adultización”). Muchas veces estas características son enseñadas explícitamente por los adultos, familia y profesorado. En ocasiones, este relato evidencia, junto con la naturalización de la violencia como medio de disciplina, la internalización por parte de los adolescentes de la responsabilidad de ser víctima de situaciones de agresividad y violencia por parte de la familia, lo que se generaliza al contexto escolar en la frase "algo habrá hecho" para que le peguen o molesten: “...es que ellos tienen más derecho...”. "Porque no le pueden devolver el golpe los hijos tampoco" (hombre, 13 años). La naturalización de la violencia y medios coercitivos, punitivos y agresivos en la interacción de los adultos con ellos, como manera de disciplinar, poner límites y enseñar a defenderse, aparecen como factores reforzadores de la violencia. Un estudiante hace una declaración bastante descriptiva al respecto: “...si los papás le enseñan (...) si los alejan de las malas juntas y se preocupan y los profesores no los dejan y ninguno de los adultos le permiten o aplauden que sea violento, el niño va a tener que dejar de ser así, porque si no a nadie le importa que otros sufran" (hombre, 12 años).

\section{Análisis Selectivo: Demanda del Rol Adulto de Afecto-Protección y Formación}

El fenómeno emergente central del estudio es la demanda por parte de los adolescentes del ejercicio del rol adulto de afecto, protección y formación (Figura 2).

El rol de afecto y protección se orienta a la necesidad de los adolescentes de que los adultos construyan un espacio afectivo caracterizado por la seguridad, contención y empatía, que los provea de una confirmación de sí mismos, los valide con las complejidades propias de su etapa del ciclo vital y les asegure un vínculo seguro, otorgándoles un sentido de pertenencia. Desde sus relatos, este vínculo afectivo les provee una sensación de seguridad y protección, los mantiene "tranquilos", porque se sienten apoyados y queridos. La demanda de formación se refiere a la necesidad que refieren los estudiantes de ser orientados y educados en recursos socioemocionales, específicamente en la capacidad de manejo emocional, control de impulsos, resolución de conflictos ("saber defenderse"), confianza en sí mismos y reflexividad. Demandan que se les enseñen tempranamente estas habilidades y se les entreguen herramientas útiles para prevenir y enfrentar la violencia escolar, "porque ahora ya no sirve de nada" (hombre, 14 años). Quienes reconocen haber contado con estos recursos vinculares y formativos refieren que les ha servido para alejarse de situaciones de agresividad y violencia: "Mi mamá me explica que meterse en peleas, violencia, no es bueno, y yo ya no tengo problemas, sé defenderme, es que depende con el apoyo de la casa, cómo educan los papás, sino los niños no llegan a ninguna parte" (hombre, 12 años). 


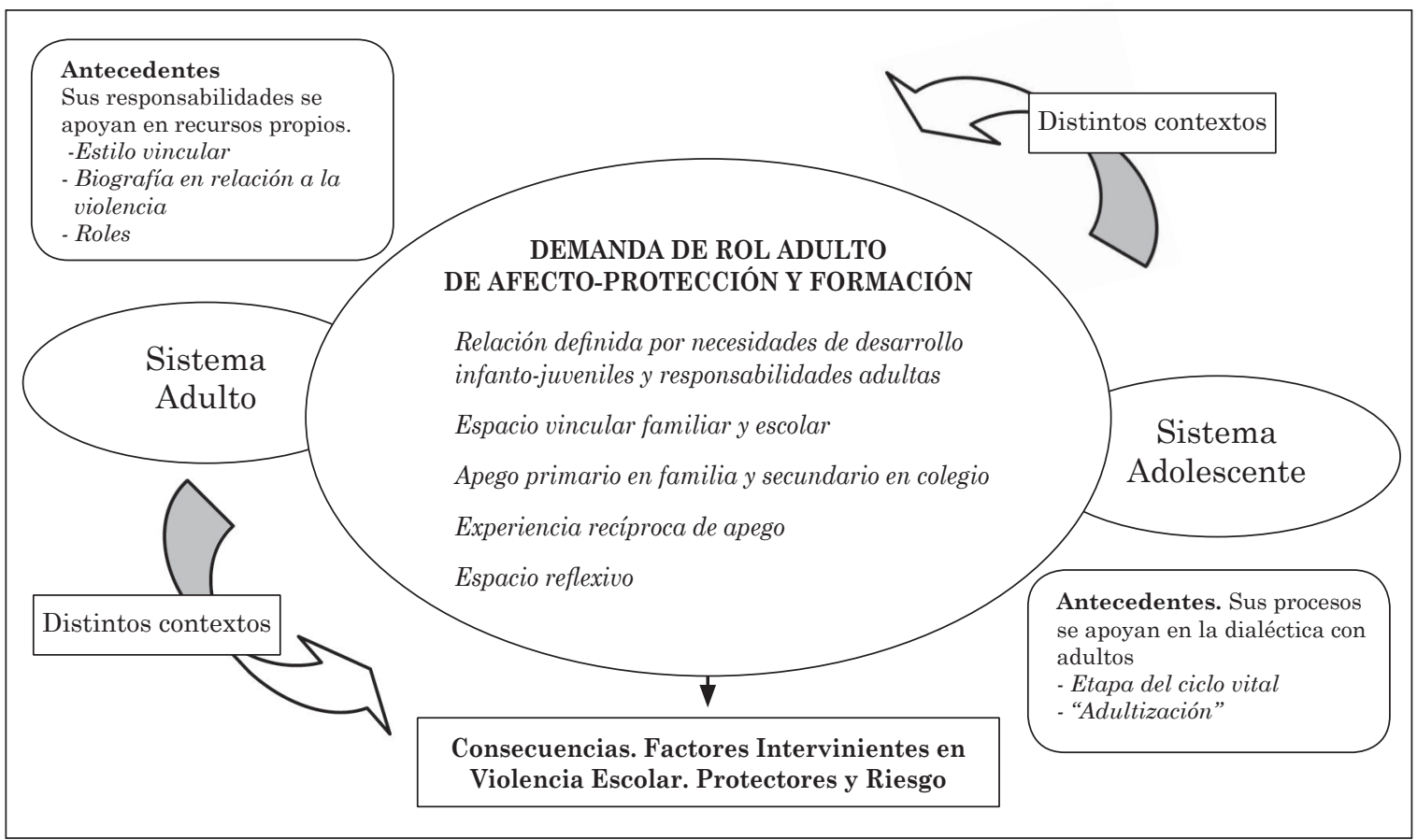

Figura 2. Demanda del rol adulto de afecto-protección y formación.

En los discursos de los adolescentes se atribuye importancia como figuras vinculares significativas a los padres y profesores, de quienes no perciben siempre un efectivo ejercicio de su rol: "Pero ustedes los grandes no saben lo que pensamos nosotros, no nos toman en cuenta" (mujer, 14 años). Cuando los padres y profesores generan o promueven interacciones afectivas, protectoras y formativas, les resulta más fácil enfrentarse a los desafíos de su etapa de desarrollo, al contar con esta protección y guía. Refieren la importancia de la recepción de cariño y de consejos para su ajuste con los pares y su medio. Para ello, la cercanía emocional, la adecuada expresión de los afectos, el cariño durante su etapa infantil, la buena comunicación, las normas claras y equitativas, la empatía con sus necesidades, la orientación y formación tempranas y permanentes en temáticas de violencia constituyen elementos inhibidores del involucramiento en violencia.

La necesidad de validarse y sentirse reconocidos como un otro legítimo, de ser querido, cuidado, protegido y formado, resultan elementos centrales en el desarrollo de la identidad. Esto aparece en el discurso adolescente como una necesidad de ser comprendidos, escuchados y apoyados. La cercanía y sintonía emocional de las figuras adultas significativas generan una sensación de pertenencia y confirmación de su sí mismo. Esta relación es recursiva, ya que también confirma y refuerza el rol parental y el de los profesores, lo que permite legitimar su autoridad. En este sentido, los límites y normas claras aparecen como primordiales a la hora de interactuar, pues ordenan la relación y las responsabilidades, permitiendo una auto-confirmación y, al mismo tiempo, la confirmación y respeto por los pensamientos y derechos del otro. Por el contrario, la invisibilización genera, según los estudiantes, una tendencia a sobre-adaptarse a las situaciones y adquirir responsabilidades adultas: “... ahora como que los padres no entienden, no saben los límites. Casi siempre esos problemas más chicos se transforman en problemas más grandes" (hombre, 13 años).

En las comprensiones de la violencia escolar de los adolescentes el rol adulto se sostiene en sus propias experiencias vinculares, sean estas armónicas o violentas (“depende si son familias, así, violentas", hombre, 11 años), en las habilidades para afrontar conflictos ("Se pelean con los vecinos", hombre, 12 años) y en las construcciones vinculares, afectivas y estables o agresivas e 
inestables. Lo mismo sucede respecto de los profesores. En el relato de los entrevistados la interacción con y en el contexto escolar surge como un espacio privilegiado para desarrollar habilidades sociales y afectivas, como el afrontamiento de conflictos, la capacidad de poner límites, el auto-cuidado y la protección por medio de normas y límites claros, justos y equitativos. En este sentido, un buen clima escolar, caracterizado por el énfasis en el entrenamiento de habilidades socioafectivas y por una buena comunicación entre profesores y estudiantes, es percibido como una necesidad en términos preventivos.

\section{Discusión}

El presente estudio tuvo por objetivo profundizar, desde una perspectiva ecológica y relacional, los factores intervinientes en la violencia escolar desde la perspectiva de los adolescentes. El discurso de los entrevistados conceptualiza la violencia escolar como un fenómeno interaccional, superando una perspectiva centrada solamente en la relación violenta directa y comprendiéndola dentro de un contexto global. Aun cuando reconocen la posibilidad de decidir involucrarse en dinámicas violentas, señalan que hay responsabilidades por lo menos compartidas con los adultos significativos, pues en general los perciben desinteresados en sus conflictivas y con dificultades para dar alternativas relacionales, no coercitivas ni violentas, frente al riesgo o desarrollo de situaciones violentas.

Los resultados confirman el carácter relacional y adaptativo de la violencia, no solo por su función en la organización de la ecología de pares, como señalan diferentes autores (Almeida, et al., 2007; Berger \& Rodkin, 2011; Bergin \& Bergin, 2009; Hawley, Little \& Rodkin, 2007), sino también por la funcionalidad adaptativa que puede cumplir en el proceso de desarrollo de los niños y en sus relaciones con los adultos significativos. La violencia es significada como una manera de posicionarse socialmente y lograr estatus grupal y popularidad (Berger \& Rodkin, 2011; García \& Madriaza, 2005). La amistad y sus códigos relacionales surgen de los discursos de los entrevistados como un elemento esencial para involucrarse o no en dinámicas de violencia. Para atreverse tan solo a molestar a otro o acceder a pelear, la mayoría refiere que resulta un elemento básico contar con amigos que lo defiendan (fianza). Esta necesidad se sustenta en el énfasis en el grupo de pares y la búsqueda de confianza. Los preadolescentes explican que rara vez la violencia se da solo entre dos individuos y, cuando esto ocurre, normalmente es un asunto que no pasa a mayores y no genera mayor daño. La mayoría de las veces la presión del grupo es determinante en la decisión de involucrarse en violencia por la necesidad de "tener grupo", que permite contar con un espacio identitario y protector.

Desde otra perspectiva, los resultados confirman la importancia del sano desarrollo vincular adolescente, es decir, relaciones positivas con los padres u otras figuras significativas, que disminuirían la ocurrencia de violencia escolar (Bergin \& Bergin, 2009; Olweus, 1997; Orpinas \& Horne, 2006). Específicamente, los resultados destacan la necesidad de reconocimiento, confirmación y diferenciación, que ha sido extensamente documentada como la base de la constitución del sí mismo y de la identidad (Benjamin, 1988; Stern, 1991). Son procesos que se basan en la interacción primaria con las figuras de apego temprano, que se van actualizando a lo largo del desarrollo, y en la etapa adolescente se configuran principalmente con los amigos, las primeras relaciones románticas y en el vínculo con los profesores, quienes, además de los padres, pasan a ser figuras de apego secundario durante el período escolar. Lo anterior supone una paradoja (Benjamin, 1988), pues cuando los preadolescentes necesitan que los padres y profesores los guíen, los sostengan y acojan, necesitan también libertad de acción en los espacios en que se mueven, especialmente en las dinámicas de pares; por esto, se refieren a la necesidad de normas y límites como un encuadre y protección de su actuar. Esta paradoja es confirmada en el relato de los adolescentes sobre la insatisfacción de sus necesidades socioafectivas desde el mundo adulto. La identidad se define como un producto de la vinculación y está determinada por formas de relación (Gergen, 1985). En la construcción de la identidad, la confirmación del sí mismo utiliza los medios disponibles para ello, ya sea a través de comportamientos constructivos y de buen trato o a través de comportamientos dañinos y violentos, como un modo de llamar 
la atención y marcar la frontera con los otros. Así, la responsabilidad de los adultos es proveer un contexto favorecedor para la construcción de identidad, a través de vínculos estables y amorosos, los cuales deben ir acompañados de una visibilización e interés real por las necesidades de los adolescentes. Tal como propone Dio Bleichmar (2004), los padres y adultos significativos deben otorgar significado relacional a la conducta de los niños y adolescentes.

En relación a lo anterior, la noción de reflexividad o mentalización (Fonagy, 2000) surge como un elemento central en la posibilidad de proteger a los niños y jóvenes de involucrarse en dinámicas de violencia. Si los adolescentes perciben una posibilidad de decidir involucrarse o no en violencia, resulta importante considerar espacios reflexivos y comprensivos de la violencia que fundamenten dicha decisión. La habilidad reflexiva o mentalización es la capacidad que habilita al sujeto para registrar e identificar sus propios sentimientos, deseos y pensamientos y discernir los motivos por los cuales surgen. La capacidad de mentalizar surge de las experiencias interpersonales tempranas, en particular, de las vividas con los objetos primarios de apego, y se actualiza durante el desarrollo, en función de las interacciones con padres, cuidadores (Fonagy, 2000) y profesores. A través de la mentalización y vínculos afectivos con otros se fortalece y desarrolla el sí mismo y la identidad, puesto que se toma conciencia de la vida emocional. Así es posible lograr una comprensión más profunda de la propia experiencia afectiva y advertir nuevos significados en los afectos, permitiendo una mayor regulación de estos. Desde el concepto de mentalización, se desprende la importancia de trabajar con profesores y padres, más que con los propios adolescentes, porque son ellos los que contienen y guían el cambio. Esto es especialmente relevante a la luz de los resultados y la demanda explícita de los adolescentes por estos espacios. La escuela se constituye en un contexto privilegiado de mentalización y apego, al actuar como un espacio potencial o área intermedia de la experiencia, situada entre la realidad psíquica interna y la realidad exterior, que facilita el desarrollo saludable a través del diálogo inter-generacional afectivo (Winnicott, 2002). Inclusive, el colegio es un contexto que, al constituirse como un espacio de apego secundario, puede facilitar la reparación de conmociones psíquicas, como son los actos violentos, al desarrollar un clima afectivo acogedor, respetuoso y empático. Ya que en el contexto sociocultural la agresividad y las estrategias coercitivas son maneras de organizarse socialmente, resulta difícil transmitir claramente a los niños y adolescentes formas nutritivas y pacíficas de relacionarse con los otros, siendo más común la tendencia a repetir estilos organizadores coercitivos y castigadores. Según sus relatos, resulta necesario un acercamiento desde los afectos y un desarrollo de sus habilidades sociales y emocionales que les den seguridad en sí mismos y en los otros. Se observa una necesidad de organización con jerarquías claras y respetuosas de los derechos propios y del otro, que se constituyan en elementos que hagan inviable la presencia de dinámicas violentas y abusivas. El contexto escolar podría constituirse, así, en un medio suficientemente bueno, como propone Winnicott (2002), adecuado a las necesidades del niño y adolescente.

Algunas limitaciones del presente estudio se constituyen en desafíos para futuras investigaciones en este tema. En primer lugar, se hacen necesarias una adecuada definición y la delimitación del fenómeno en estudio, considerando la amplia confusión existente en torno a la violencia escolar y fenómenos mediáticos, como el bullying. Por otra parte, algunos desafíos metodológicos implican la posibilidad de utilizar un muestreo teórico hasta la saturación de la información, incluyendo otros grupos etarios y contextos escolares, la utilización de múltiples fuentes de reporte (profesores y padres) y el cruce de dicha información, integrando una perspectiva de género. Por último, debe considerarse la posibilidad de complementar estos resultados con datos cuantitativos que permitan dimensionar los resultados y conclusiones expuestas.

No obstante lo anterior, la consideración de la perspectiva de los adolescentes en cuanto actores de la violencia escolar entrega importantes elementos para su comprensión, conceptualización y lineamientos para la prevención y permite superar perspectivas que no contemplan la subjetividad de los estudiantes y los sentidos que estos otorgan a su experiencia cotidiana. Los resultados de este estudio resaltan la necesidad y demanda explícita de los adolescentes por comprender y manejar el fenómeno de la violencia escolar. 


\section{Referencias}

Almeida, A., Lisboa, C. \& Caurcel, M. J. (2007). ¿Por qué ocurren los malos tratos entre iguales?: explicaciones causales de adolescentes portugueses y brasileños. Revista Interamericana de Psicología, 41, 107-118.

Benjamin, J. (1988). Los lazos de amor: psicoanálisis, feminismo y el problema de la dominación (1ª ed.). Buenos Aires, Argentina: Paidós.

Berger, C., Karimpour, R. \& Rodkin, P. C. (2008). Bullies and victims at school: Perspectives and strategies for primary prevention. En T. W. Miller (Ed.), School violence and primary prevention (pp. 295-322). New York, NY: Springer.

Berger, C. \& Lisboa, C. (2008). Hacia una comprensión ecológica de la agresividad entre pares en el microsistema escolar. En C. Berger \& C. Lisboa (Eds.), Violencia escolar: estudios y posibilidades de intervención en Latinoamérica (pp. 59-83). Santiago, Chile: Editorial Universitaria.

Berger, C. \& Rodkin, P. C. (2009). Male and female victims of male bullies: Social status differences by gender and informant source. Sex Roles, 61, 72-84. doi:10.1007/s11199-009-9605-9

Berger, C. \& Rodkin, P.C. (2011), Group Influences on Individual Aggression and Prosociality: Early Adolescents Who Change Peer Affiliations. Social Development. doi:10.1111/j.1467-9507.2011.00628.x

Bergin, C. \& Bergin, D. (2009). Attachment in the classroom. Educational Psychology Review, 21, 141-170. doi:10.1007/ s10648-009-9104-0

Bowlby, J. (1999). Vínculos afectivos: formación, desarrollo y pérdida (3ª ed.). Madrid, España: Ediciones Morata.

Bronfenbrenner, U. (1979). The ecology of human development: Experiments by nature and design. Cambridge, MA: Harvard University Press.

Chile, Ministerio del Interior. (2011). Tercera Encuesta Nacional de Violencia en el Ámbito Escolar 2009. Santiago, Chile: Autor, División de Seguridad Pública. Extraído de http://www.seguridadpublica.gov.cl/files/presentacion_ violencia_escolar_2009_web.pdf

Contador, M. (2001). Percepción de la violencia escolar en estudiantes de enseñanza media. Psykhe, 10(1), 69-80.

Del Barrio, C., Almeida, A., van der Meulen, K., Barrios, A. \& Gutiérrez, H. (2003). Representaciones acerca del maltrato entre iguales, atribuciones emocionales y percepción de estrategias de cambio a partir de un instrumento narrativo: SCAN-Bullying. Infancia y Aprendizaje, 26, 63-78.

Del Barrio, C., Martín, E., Almeida, A. \& Barrios, A. (2003). Del maltrato y otros conceptos relacionados con la agresión entre escolares, y su estudio psicológico. Infancia y Aprendizaje, 26, 9-24.

Dio Bleichmar, E. (2004). Modelos interactivos entre la genética de la conducta y la parentalización. Aperturas Psicoanalíticas: Revista de Psicoanálisis, 17, artículo 5. Extraído de http://www.aperturas.org/articulos.php?id=0 000298\&a=Estudios-sobre-la-relacion-herencia-ambiente-en-la-temprana-infancia

Espelage, D. L., Holt, M. K. \& Henkel, R. R. (2003). Examination of peer-group contextual effects on aggression during early adolescence. Child Development, 74, 205-220. doi:10.1111/1467-8624.00531

Fonagy, P. (2000). Apegos patológicos y acción terapéutica. Aperturas Psicoanalíticas: Revista de Psicoanálisis, 4 , artículo 4. Extraído de http://www.aperturas.org/articulos.php?id=0000104\&a=Apegos-patologicos-y-accionterapeutica

Gallese, V., Eagle, N. M. \& Migone, P. (2007). Intentional attunement: Mirror neurons and the neural underpinnings of interpersonal relations. Journal of the American Psychoanalytic Association, 55, 131-175. doi:10.1177/000306 51070550010601

García, M. \& Madriaza, P. (2005). Sentido y sinsentido de la violencia escolar: análisis cualitativo del discurso de estudiantes chilenos. Psykhe, 14(1), 165-180. doi:10.4067/S0718-22282005000100013

Gergen, K. J. (1985). The social constructionist movement in modern psychology. American Psychologist, 40, 266-275. doi:10.1037/0003-066X.40.3.266

Glaser, B. G. \& Strauss, A. L. (1967). The discovery of grounded theory: Strategies for qualitative research. Chicago, IL: Aldine.

Graham, S. \& Juvonen, J. (1998). Self-blame and peer victimization in middle school: An attributional analysis. Developmental Psychology, 34, 587-599. doi:10.1037/0012-1649.34.3.587

Hawley, P. H., Little, T. D. \& Rodkin, P. C. (Eds.) (2007). Aggression and adaptation: The bright side to bad behavior. Mahwah, NJ: Lawrence Erlbaum.

Jimerson, S. R., Swearer, S. M. \& Espelage, D. L. (Eds.) (2010). Handbook of bullying in schools: An international perspective. New York, NY: Routledge.

Olweus, D. (1997). Conductas de acoso y amenaza entre escolares. Madrid, España: Ediciones Morata.

Organización Panamericana de la Salud \& Ministerio Federal de Cooperación Económica y Desarrollo-GTZ (2008). iPreparados, listos, ya: una síntesis de intervenciones efectivas para la prevención de violencia que afecta a adolescentes y jóvenes. Washington, DC: Organización Panamericana de la Salud.

Orpinas, P. \& Horne A. M. (2006). Bullying prevention: Creating a positive school climate and developing social competence. Washington, DC: American Psychological Association. doi:10.1037/11330-000

Roisman, G. I., Padrón, E., Sroufe, L. A. \& Egeland, B. (2002). Earned-secure attachment status in retrospect and prospect. Child Development, 73, 1204-1219. doi:10.1111/1467-8624.00467

Scharf, M. \& Mayseless, O. (2007). Putting eggs in more than one basket: A new look at developmental processes of attachment in adolescence. New Directions for Child and Adolescent Development, 117, 1-22. doi:10.1002/cd.191

Shapiro, E. R. (1991). Cambio individual y desarrollo familiar: la individuación como proceso familiar. En C. J. Falicov, Transiciones de la familia (pp. 231-259). Buenos Aires, Argentina: Amorrortu. 
Smith, P. K., Cowie, H., Olafsson, R. F. \& Liefooghe, A. P. D. (2002). Definitions of bullying: A comparison of terms used, and age and gender differences, in a fourteen-country international comparison. Child Development, 73, 1119-1133. doi:10.1111/1467-8624.00461

Stern, D. N. (1991). El mundo interpersonal del infante. Buenos Aires, Argentina: Paidós.

Strauss, A. \& Corbin, J. (2002). Bases de la investigación cualitativa: técnicas y procedimientos para desarrollar la teoría fundamentada. Medellín, Colombia: Editorial Universidad de Antioquia.

Sullivan, K., Cleary, M. \& Sullivan, G. (2005). Bullying en la enseñanza secundaria. El acoso escolar: cómo se presenta y cómo afrontarlo. Barcelona, España: Ediciones Ceac.

Troop-Gordon, W. \& Ladd, G. W. (2005). Trajectories of peer victimization and perceptions of the self and schoolmates: Precursors to internalizing and externalizing problems. Child Development, 76, 1072-1091. doi:10.1111/j.14678624.2005.00898.x

Winnicott, D. (2002). Preocupación maternal primaria. En D. Winnicott, Escritos de pediatría y psicoanálisis (pp. 405412). Buenos Aires, Argentina: Paidós.

Fecha de recepción: Marzo de 2011.

Fecha de aceptación: Agosto de 2011. 